\title{
SonicSpray: A Technique to Reconfigure Permeable Mid-Air Displays
}

\author{
Mohd Adili Norasikin ${ }^{12}$ \\ ${ }^{1}$ Pervasive Computing \& Educational Technology \\ Center for Advanced Computing Technology \\ Fakulti Teknologi Maklumat dan Komunikasi \\ Universiti Teknikal Malaysia Melaka \\ adili@utem.edu.my
}

\author{
Diego Martinez Plasencia ${ }^{2}$, Gianluca Memoli², \\ Sriram Subramanian ${ }^{2}$ \\ ${ }^{2}$ School of Engineering and Informatics \\ University of Sussex \\ \{m.norasikin, dm372, g.memoli, \\ sriram\}@sussex.ac.uk
}
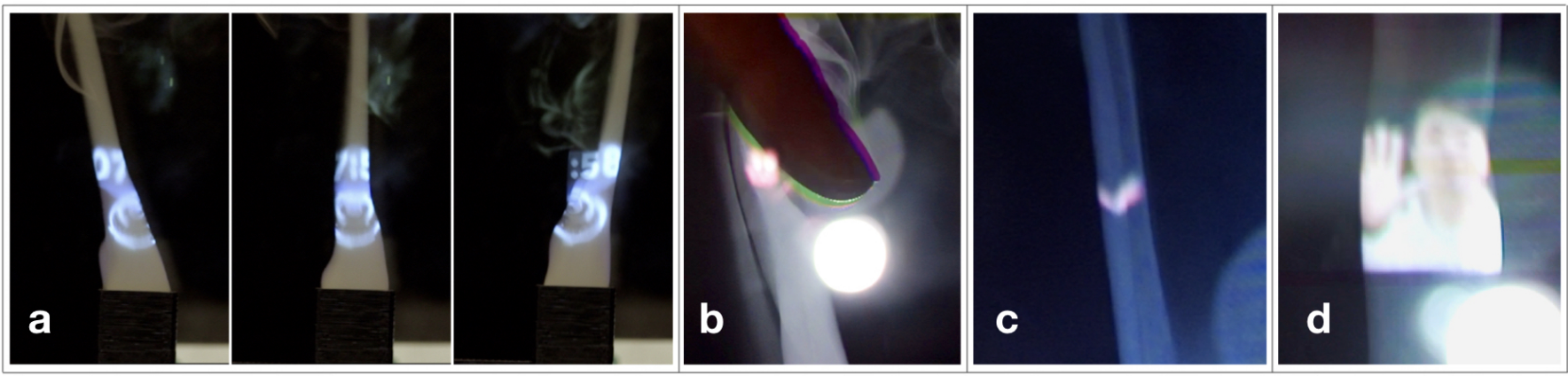

Figure 1. Applications enabled by SonicSpray: (a) SonicSpray is visualising a graphical clock in mid-air by laterally oscillating its narrow mist, (b) game of whack a mole, (c) projection of butterfly that is moving in 3D spatial space, (d) a visual of a person in a video call application.

\begin{abstract}
Permeable, mid-air displays, such as those using fog or water mist are limited by our ability to shape and control the aerosol and deal with two major issues: (1) the size and complexity of the system, and (2) the creation of laminar flow, to retain display quality. Here we present SonicSpray, a technique using ultrasonic Bessel beams to create reconfigurable mid-air displays. We build a prototype from low-cost, off-the-shelf parts. We explore the potential and limitations of SonicSpray to create and redirect laminar flows of fog. We demonstrate a working prototype that precisely controls laminar aerosols through only $6 \times 6$ ultrasound transducers array. We describe the implementation steps to build the device, verify the control and projection algorithm for the display, and evaluate its performance. We finally report our exploration of several useful applications, in learning, entertainment and arts.
\end{abstract}

\section{Author Keywords}

permeable mid-air display; reconfigurable mid-air displays; ultrasonic control

Permission to make digital or hard copies of all or part of this work for personal or classroom use is granted without fee provided that copies are not made or distributed for profit or commercial advantage and that copies bear this notice and the full citation on the first page. Copyrights for components of this work owned by others than ACM must be honored. Abstracting with credit is permitted. To copy otherwise, or republish, to post on servers or to redistribute to lists, requires prior specific permission and/or a fee. Request permissions from permissions@acm.org.

ISS '19, November 10-13, 2019, Deajon, Republic of Korea.

Copyright () 2019 Association of Computing Machinery.

ACM ISBN 978-1-4503-6891-9/19/11 _..\$15.00.

http://dx.doi.org/10.1145/3343055.3359704

\section{CCS Concepts}

-Hardware $\rightarrow$ Displays and imagers; Touch screens;

\section{INTRODUCTION}

Imagine a display that is shapeless as water, being able to reconfigure without physical contact, interactive and computationally reconfigurable to form shapes in mid-air. This type of display can be seen in science fiction movies, for instance, Iron Man and today these mid-air displays have been demonstrated through many proofs of concepts, for example, PixieDust [19], HoloVect [28], MistForm [35], and Luciola [25]. These type of displays consistently draw the attention of the imaginative community, mainly in interactive graphics on Human-Computer Interaction (HCI).

Despite the technology advancements in these permeable, reconfigurable, mid-air displays, they usually involve a high price point (acknowledged as one main issue towards their commercialization[33]) and remain bulky [19, 29, 13, 25]. The ability to provide fine resolution in their reconfiguration while retaining a minimal form factor is acknowledged as another main challenge [2]. This challenge is even more crucial for permeable displays dealing with aerosols, where the ability to control the shape and trajectory of aerosols in mid-air, while retaining a laminar flow is acknowledged as a factor limiting their potential form factors [29]. As a result, existing permeable, reconfigurable, mid-air displays are hardly found in minimal form factors, and this comes at the expense of them not being reconfigurable [27, 34, 1], not being portable [6], or both [4, 23]. 
As a key contribution, this paper describes SonicSpray, a novel technique to reconfigure permeable mid-air displays using ultrasound Bessel beams (concept in Figure 10(a)). SonicSpray is developed around two key properties: 1) it enables mid-air display control through a minimal form factor [2], built using low cost, off-the-shelf components [33]; and 2) it maintains laminarity of the flow during reconfiguration, to retain display quality [26]. These properties enable four key advantages: 1) it removes enclosures (i.e., transparent electrodes) like those in [29]; 2) it is silent, avoiding fans or other noisy moving parts [35]; 3) it allows simple and precise real-time control of the laminar airflow, using only one control point on XY-plane; and 4 ) it has a minimal form factor. We leverage these benefits for our technique, enabling the creation of novel mid-air displays, which we explore in the paper. Our technique can also be combined with other existing techniques (e.g., [27, 34, $29,12])$ to enable further form factors.

We first describe our SonicSpray technique, which uses an elongated Bessel beam to create laminar aerosol flows, and diffraction gradients to allow their real-time reconfiguration. The technique is compatible with phased arrays of transducers (PATs) and hybrid modulators of metamaterials (MM) [18] and, in all cases, the direction of the flow can be easily and precisely controlled by redirecting the beam towards a user-defined control point. We then explore the potential of the SonicSpray technique (beyond the prototype in Figure 1(a)) in two steps: First, we describe the principle and explore the effects of varying PAT sizes (from $16 \times 16$ to $2 \times 2$ transducers) identifying minimum operational form factors for SonicSpray, based on sound field simulations (i.e., COMSOL Multiphysics), wind speed test, and laminar airflow tests. Second, we verify the spatial control algorithm of the proposed technique with careful analysis of the sound field in COMSOL Multiphysics and evaluating it in terms of lateral oscillation (i.e., reported as the biggest issue for mid-air displays [14]). Particularly, we study the effects of lateral oscillation on the quality of the laminar aerosol, as well as the effects of varying the number of transducers used. Finally, we demonstrate some of the working applications enabled by the SonicSpray, where we demonstrate the ability of SonicSpray to create and accurately direct aerosols while retaining laminarity of the flow required for display purposes. We finish with a discussion on the limitations of SonicSpray, and also on the novel designs that it enables, both as a stand-alone control technique, or in combination with other existing techniques.

\section{RELATED WORK}

This section reviews two groups of reconfigurable mid-air displays in HCI: permeable and impermeable, according to the challenges identified above: their ability for mid-air aerosol control [29], to retain laminar flows [26], as well as small form factors and precise control [2].

\section{Impermeable Mid-Air Displays}

There are many techniques to reconfigure impermeable mid-air displays. SensaBubble [32] used computer-controlled fans to direct a fog-filled bubble, only allowing very coarse control on the bubble's trajectory. Sahoo et al. [29] improved trajectory control of the fog-filled bubble using electric fields created by an array of high-voltage transparent electrodes. This improvement in control came at the expense of hindering user interaction (i.e., presence of electrodes, high voltages) and, in both cases, the bubble popped when touched.

Levitation approaches have also been explored. ZeroN [14] presented a mid-air display using magnetic levitation and projection mapping, but the system was bulky and costly, requiring a combination of two systems to control the levitated ball (magnetic for vertical position, mechanical for lateral displacements). Aerial Tunes [3] used Bernoulli's effect to control mid-air displays on a vertical axis, and Flotia [36] improved such control, again by including a second mechanical system.

Other works create reconfigurable mid-air displays, using ultrasonic levitation and PAT arrays (e.g. 16x16 transducers). Pixie Dust [19] used four PATs and demonstrated the manipulation of objects of different material and density. Other works used opposed PATs [25] or a single PAT with a flat reflector [10] and have been used to demonstrate precise $3 \mathrm{D}$ positioning of particles in space [21], or rotations and multi-point levitation [16]. This technique has also been implemented for small form factors. For instance, JOLED [30] used 30mmx $80 \mathrm{~mm}$ PAT and demonstrated control on a bi-stable display in midair, by coating the voxels with titanium dioxide $\left(\mathrm{TiO}^{2}\right)$ and using electric fields to flip them. Point-and-Shake [6] used $80 \mathrm{mmx} 40 \mathrm{~mm}$ PATs to create and select mid-air buttons. FloatingCharts [22] created mid-air point charts, reconfigured in real-time through compositions of smaller PATs, designed in a round shape (PAT $\varnothing 30 \mathrm{~mm}$ ). However, in all these cases, displays are made of sparse particles, and physical touch will eventually disturb the stability of these particles [6]. This issue will limit their use as interactive display systems.

\section{Permeable Mid-Air Displays}

Permeable displays allow users to reach and interact inside the display volume, reconstructing themselves around the user's hands/bodies. However, this involves the use of free-flowing aerosols, making their control more challenging and limiting the number of existing techniques and form factors.

The most prominent technique is FogScreens [26, 27], which uses fans to create a laminar flow but results in permeable displays constrained to a flat form factor. Works in $[13,11]$ showed a volumetric mid-air display by using a 2D array of static fog nozzles, or nozzles being mechanically actuated [12], to adjust the depth of parts of the display. This concept was extended to a larger form-factor by MistForm [35], combining mechanical actuators and fog nozzles mounted on a flexible PVC pipe, achieving an adaptive shape-changing display fog screen. However, the use of fans to achieve laminar flows resulted in noise and the direction of the aerosol flow could not be changed once it left the nozzle (it only floats upwards, while SonicSpray allows mid-air redirection). In the case of MistForm, the form factor was also bulky.

Instead of using mechanical actuators, Sahoo et al. [29] demonstrated control of the mid-air direction of the fog screen (like our SonicSpray) using high-voltage electric fields but constraining the display between transparent electrodes. Gushed Diffusers [34] and BreathScreen [1] demonstrate portable form 
factors, but they still do not allow mid-air reconfiguration and their displays do not retain a laminar flow. Similarly, Hasegawa et al. [8] demonstrated mid-air control of the direction of an aerosol (for smell delivery) using PATs, but unlike SonicSpray, they do not deal with the laminar properties of the aerosol (not required for smell delivery). Also, while they acknowledge the issue of 'ghost images' which limits the control of the fog, we show how this issue is connected to the resolution of the modulator used and how the use of a hybrid modulator (i.e., a combination of a MM and PAT [18]) can avoid it, while still allowing the creation and reconfiguration of Bessel beams and retaining a laminar airflow of particles.

\section{SONICSPRAY: RECONFIGURATION TECHNIQUE}

This section describes the basic technique to create and redirect ultrasound Bessel beams and illustrates its behaviour using COMSOL simulations. Actual experiments with a real setup are described in the next section.

\section{Basic Bessel beam creation}

In this section we explain our technique, which is compatible with both PATs and hybrid modulators, and uses Bessel beams to create a stream of air particles (i.e., move the aerosol), redirect it in real-time (i.e., allow mid-air control), and to retain the laminarity of the flow (i.e., for display quality purposes). We refer to a related work by Hasegawa et al. [7] to construct the Bessel beam. In general, one can produce this Bessel beam with a conical arrangement of sound sources, where the sound waves will converge and concentrate its energy (i.e., ultrasonic radiation force) as in Figure 2(left).

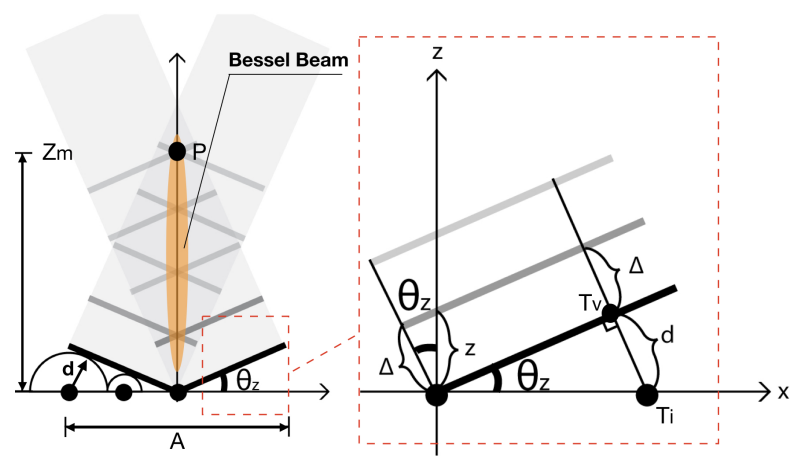

Figure 2. Geometrical Representation of Bessel Beam: left) An elongated and slim sound beam (i.e., Bessel beam) is created as a result of a conical and propagated wavefronts, and right) Information needed to create the illustrated wavefront from a transducer in a Phased Array Transducer (PAT).

To construct the beam, we use a modulator (PAT or MM), operating at an ultrasonic frequency $(f=40 \mathrm{kHz})$ in air (speed of sound $c=343 \mathrm{~ms}^{-1}$ ). The algorithm includes two steps (see Figure 2(right)): Firstly, we compute the angle $\theta_{z}$ in Equation (1), given a constant aperture A (modulator diameter), and $z_{m}$ as the maximum height of the beam.

$$
\theta_{z}=\tan ^{-1}\left(\frac{A}{2 \cdot z_{m}}\right)
$$

Secondly, we compute the phase for each element in our modulator as in Equation (2), where $k=2 \pi / \lambda$ is the ultrasound wavenumber, and $\lambda=c / f$ is the wavelength, in our case $8.6 \mathrm{~mm}$, and $d\left(T_{i}, 0\right)$ is Euclidean distance function. The phase profile $\phi_{i}$ of the element can be electronically delivered to a PAT, or fabricated into a MM as in [18].

$$
\phi_{i}=-k \cdot d\left(T_{i}, 0\right) \cdot \sin \theta_{z}
$$

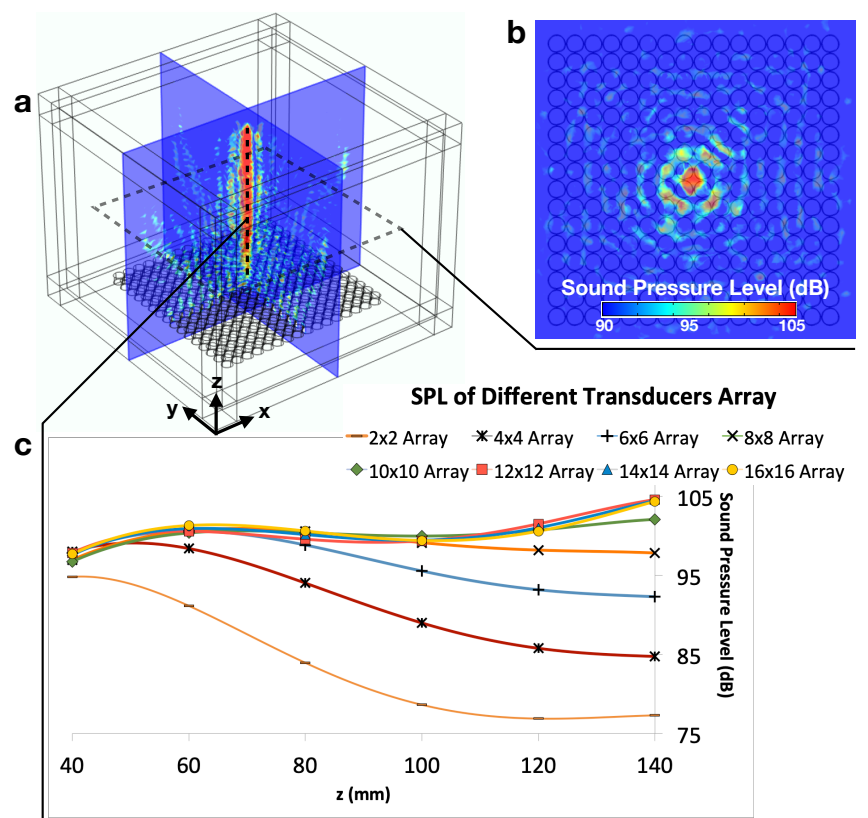

Figure 3. Simulation of Bessel Beam using COMSOL Multiphysics: (a) 3D simulation of Bessel beam sound field, (b) A horizontal slice of Bessel beam at $z=100 \mathrm{~mm}$, (c) SPL levels achieved through a different number of transducers

Figure 3 shows a 3D simulation of an example Bessel beam, created on a 16x16 PAT. The field presents an elongated beam in the middle of the array, while Figure 3(b) shows a horizontal slice $z=100 \mathrm{~mm}$, showing good focusing of the beam. Figure 3(c) shows the SPL levels achieved by varying the number of transducers in the PAT, revealing a decline in SPL as the number of transducers (i.e., sound energy) decreases, illustrating the trade-offs between PAT size, SPL and, in turn, the feasibility of reduced PAT setups to reconfigure aerosols.

\section{Lateral reconfiguration of the basic beam}

We use diffraction gratings into our method to reconfigure our Bessel beam (i.e., direct the display to the sides). This method is as alternative to the one described by Hasegawa et al. [7], typically used for optical tweezers [31,24] and it simplifies the integration of our approach with simple PATs or hybrid modulators [18] as we will illustrate here.

Given the reference point of our Bessel beam $P\left(0,0, z_{s}\right)$ (e.g., see Figure 4(A1) where $\left.z_{s}=60 \mathrm{~mm}\right)$, and a desired displacement of the Bessel beam in the $X Y$-plane $(\Delta x, \Delta y)$, we identify the position of each $i$-th element in our modulator $T_{i}\left(x_{i}, y_{i}, z_{i}\right)$. We compute the diffraction gratings phase $\psi_{i}$ for the $i$-th element $T_{i}$ by using Equation (3). In the case of a simple PAT, the final phase delay $\phi_{i}^{\prime}$ for each transducer is simply computed as 
A
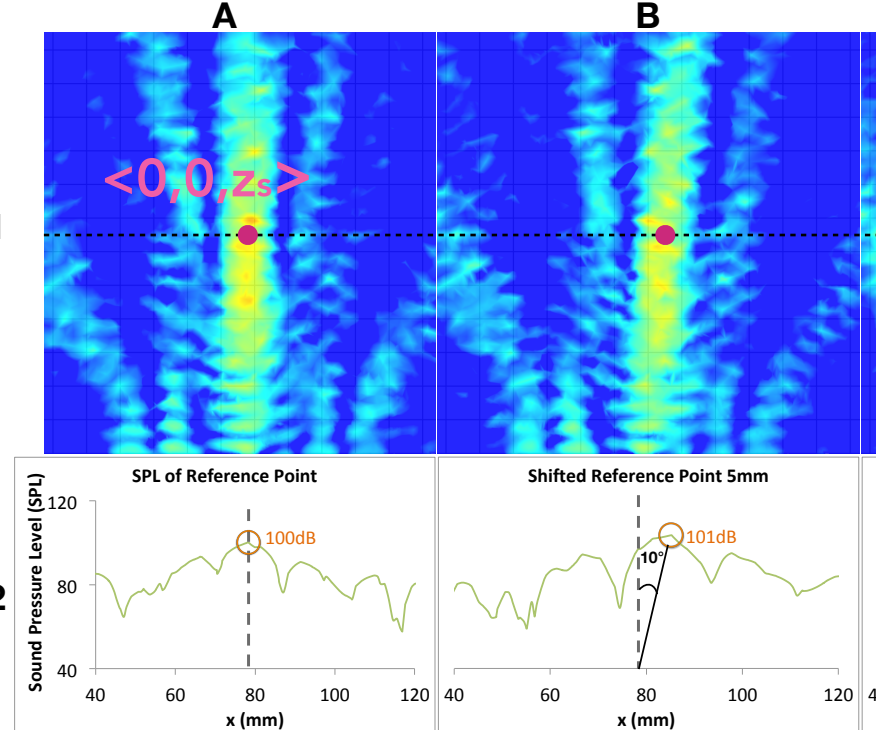

Shifted Reference Point $5 \mathrm{~mm}$

Shifted Refe

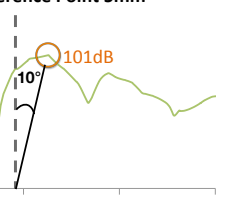

60

$x(\mathrm{~mm})$
C

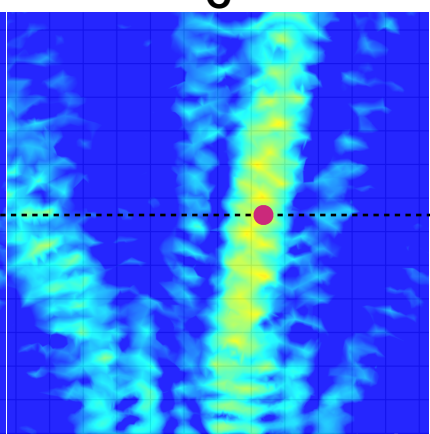

Shifted Reference Point 10mm

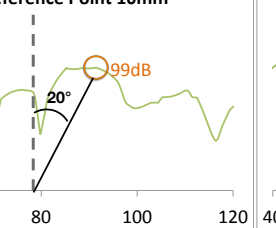

$x(\mathrm{~mm})$
D

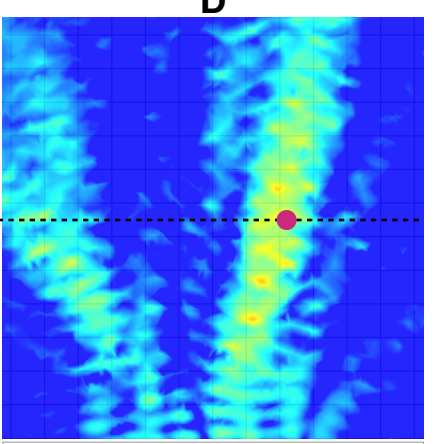

Shifted Reference Point 15mm

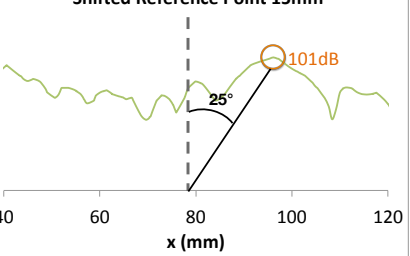

120
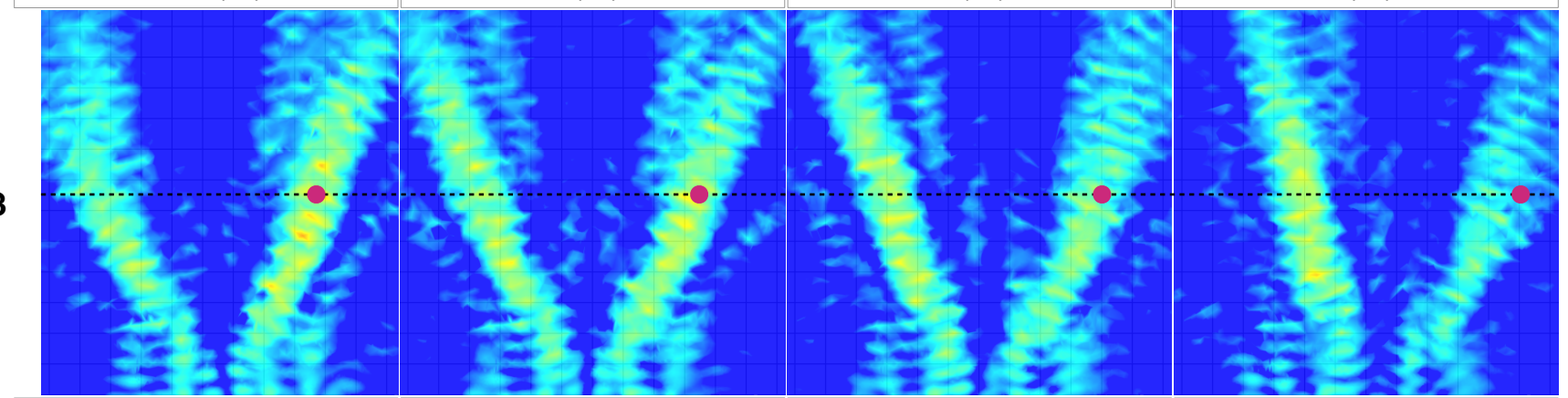

Shifted Reference Point 35mm

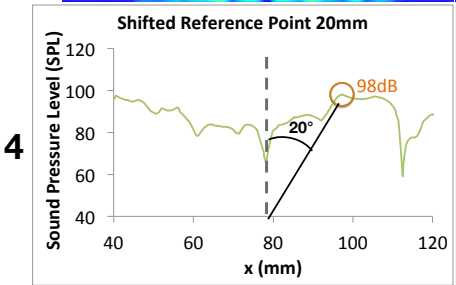

Shifted Reference Point 25mm
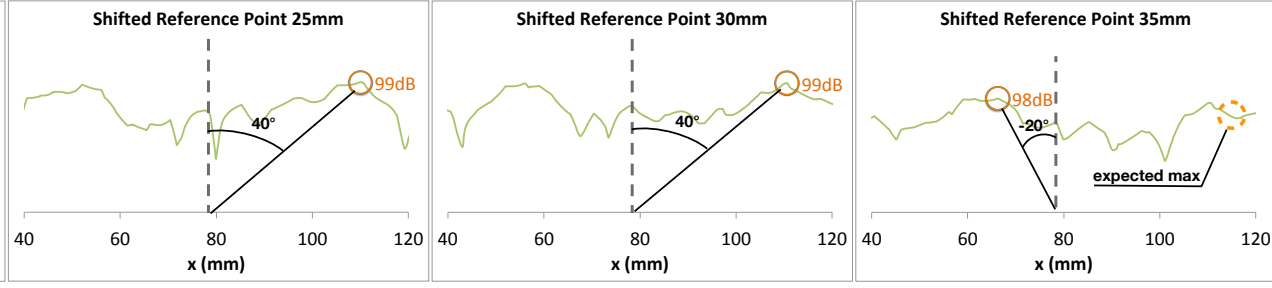

Sound Pressure Level (dB)

Reference Point - - Initial z-axis of Reference Point (60mm) " Initial x-axis of Reference Point (78mm) OMaximum SPL

Figure 4. Simulation of Beam Control Algorithm: Row (1) and (3) are sound fields of a different lateral shift, and row (2) and (4) are graphs of Sound Pressure Level (SPL) of the sound fields.

the phase addition of the Bessel beam and diffraction gratings $\left(\phi_{i}^{\prime}=\phi_{i}+\psi_{i}\right)$. Hybrid modulators will instead use $\psi_{i}$ to drive the PAT (i.e., $\phi_{i}$ is encoded in the MM).

$$
\psi_{i}=\frac{2 \pi}{\lambda \cdot z_{s}}\left(x_{i} \cdot \Delta x+y_{i} \cdot \Delta y\right)
$$

\section{Simulation of Lateral Reconfiguration Algorithm}

We explored the capabilities of our reconfiguration algorithm (COMSOL Multiphysics simulations), to laterally shift the reference point ' $P$ ', highlighting important relationships between the maximum shifting and the type of modulators used.

Figure 4 shows the simulation results of our algorithm when using PATs. Row(1) and row(3) are the sound fields of different lateral shifts on $X$-axis $(\Delta x)$, while row(2) and row(4) are cutline graphs of the sound fields at $z_{s}=60 \mathrm{~mm}$. To easily note the changes, first, we denote the initial $Z$-axis of the reference point $z_{s}$ with horizontal dashed-line, as in row(1) and (3), and initial $X$-axis with a vertical line, as in row(2) and (4). Then we denote their maximum SPL with a circle, and we identify their angle from its initial $X$-axis line. The results verified that the algorithm is able to precisely shifts our reference point ' $P$ '.

The figure shows a 'ghost' beam in our sound fields [7, 8], appearing to the left side of our intended beams in Figure 4(A3-D3). The intensity of the 'ghost' (SPL level) is low from $\Delta x=0 \mathrm{~mm}$ to $15 \mathrm{~mm}$, growing at larger shifts, until it supersedes the SPL of the main beam at $\Delta x=35 \mathrm{~mm}$ (see Figure 4(D3) and (D4)). This will result in unintended airflow when the beam is laterally shifted more than $15 \mathrm{~mm}$, an inherent limit related to the large size of PATs transducers $(10 \mathrm{~mm} \emptyset)$ [18]. 
This limitation can be solved with hybrid modulators, particularly those using small cell designs. We reused the design by Memoli et al. [17] (bricks size $\lambda / 2$ as in Figure 5(b)) to create higher quality Bessel beams. Figure 5(a) and (c) shows a comparison of the lateral shifted beam at $\Delta x=35 \mathrm{~mm}$ with PAT and with a hybrid setup (i.e., MM and PAT) respectively.

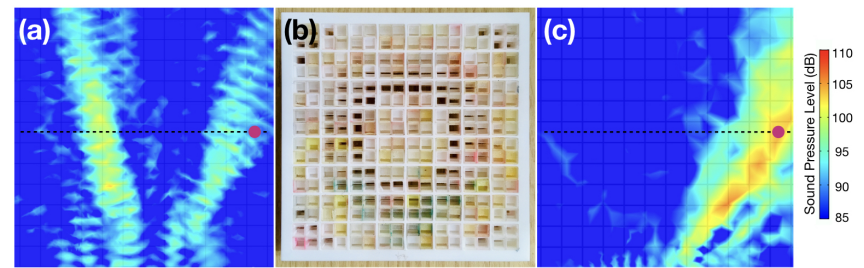

Figure 5. Comparison of approaches (bead shifted $\Delta x=35 \mathrm{~mm}$ ): (a) A PAT (transducer diameter $10 \mathrm{~mm} \varnothing$ ) fails to steer the beam according to the control point, with 'ghost' beams appearing on the left side (superseding the real beam on the right); (b)Equivalent metamaterial, using a smaller cell size $(\lambda / 2)$, and (c) resulting field using the hybrid setup (i.e., MM and PAT) and avoiding 'ghost' beams.

\section{EXPERIMENTAL CHARACTERIZATION}

Once our technique has been described, the current section describes experiments and analysis conducted to explore if SonicSpray can be used in practice to direct and control the aerosol flow, while retaining the laminarity of the flow. The relationships between the number of transducers used, the SPL levels achieved, and the resulting wind speed is analysed here (and always constrained to the laminar regime). Finally, we limit the exploration to lateral displacements of up to $35 \mathrm{~mm}$, as a way to derive results that are useful, independently of the type of modulator used (PAT or hybrid).

\section{Experimental Setup}

We assembled our prototype as in Figure 7(a). We used an offthe-shelf atomiser (made by IMECIG) and an air pump (made by SIMILK) operated at $0.75 \mathrm{~W}$. We used propylene glycol and glycerol liquid as for the mist. We used a 3D-printed squared $\left(40 \mathrm{~mm}^{2}\right)$ orifice emitter. The reason we choose squared design orifice is to minimise the number of transducers that are blocked by our emitter. We then fill a fine metal mesh in the orifice to reduce airflow velocity from the air pump, create an even distribution of mist particle over the orifice area. We carefully aligned the emitter with the expected Bessel beam to ensure the mist responds quickly to the ultrasonic radiation force. Also, the resulting emitter provided near-zero initial speed to the flow (i.e., the mist would float upwards due to buoyancy, but speed was below the minimum threshold measurable by our anemometer), ensuring that our tests only measure particle accelerations induced by our SonicSpray technique, as a result of acoustic pressure. The 16x16 PAT followed the design in [15], using transducers from Manorshi Electronics (MSO-P1040H07T, $\varnothing=10 \mathrm{~mm}$ ) operated at $40 \mathrm{kHz}$ frequency, MOSFETs to amplify control signals to $15 \mathrm{Vpp}$ (MOSFET, Microchip MIC4127) and a FPGA to control them (Cyclone IV EP4CE6). We connect the array to a PC via UART protocol at 250kbauds ( $\sim 250$ s per updates).

We included a projector-camera system, providing a simple embodiment for a mid-air display using SonicSpray, which

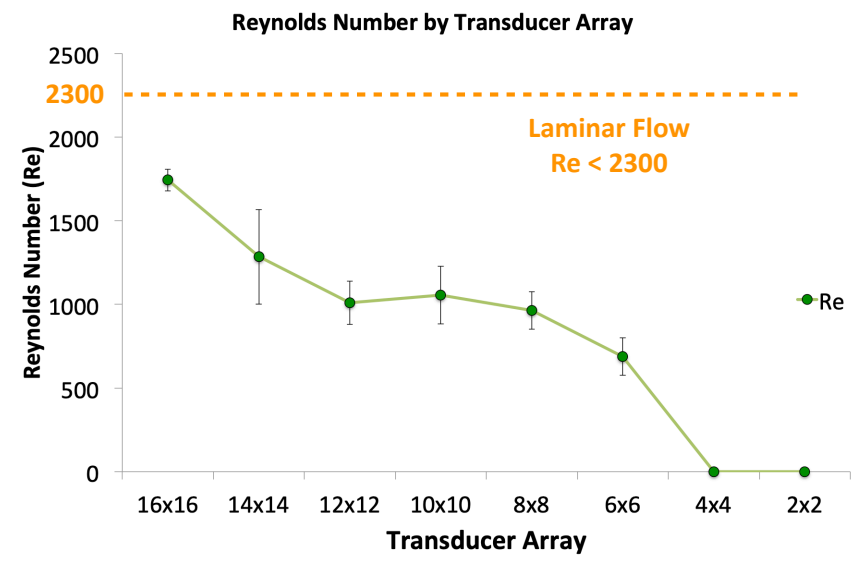

Figure 6. Graph of Reynolds Number (Re) by a different number of transducers. The threshold value for laminar is below 2300 , while turbulent is above 2300 .

we used for our application examples. The system combines a Hercules HD Twist camera (resolution of $1024 \times 768$ ), and an iCODIS LED mini projector (resolution of 854 x 480). Details of the camera-projector calibration procedure and operating software can be found in the supplementary material.

\section{Speed and Laminar Airflow Test}

To identify whether the Bessel beam produces a laminar or turbulent airflow, we calculated the ratio between mist inertial forces and its viscous forces, known as Reynolds Number $(R e)$. The $R e$ is described in Equation (4) where $v$ is the airflow speed $\left(\mathrm{ms}^{-1}\right) ; L$ is our mist outlet dimension, $0.0138 \mathrm{~m}^{2}$; and $v$ is the kinematic viscosity of the air, in our case $10^{-6} \mathrm{~m}^{2} / \mathrm{s}$. Low $R e$ number produces a smooth or laminar type of flow due to dominant viscous forces, while high $R e$ number produces turbulent flow due to dominant inertial forces. According to Reynolds Number, laminar flow occurs at $R e<2300$, and turbulent flow occurs at $\operatorname{Re}>2900$.

$$
R e=v L / v
$$

We measured the $R e$ in three steps: Firstly, we emitted the mist, and turned on the transducers array (e.g., 16x16). Secondly, We measured the produced airspeed with an anemometer (Kestrel1000) at several z-axis positions above the emitter outlet. Thirdly, we computed the $R e$ and their error.

We repeated the three steps above using a different number of transducers, and we compare the results. Figure 6 shows that $R e$ numbers for all cases are lower than 2300, showing the ability of SonicSpray to create laminar aerosol flows. Solutions with $4 \times 4$ and $2 \times 2$ transducers failed to induce airflows (i.e., speed $\sim 0 \mathrm{~m} / \mathrm{s}$ ). Figure 7(b-d) demonstrates the laminar aerosol created by remaining configurations, as well as their ability to shift the beam to the left or right as in Figure 7(b) and (d) respectively. The path followed by aerosol particles is related to the particle sizes, with ultrasonic radiation dominating the motion of larger particles $(\varnothing=5.0 \mu \mathrm{m})$, and Stokes drag [5] dominating the motion of smaller ones $(\varnothing=0.5 \mu \mathrm{m})$. As such, filtering techniques like those suggested in [29] can help improve flow control. 

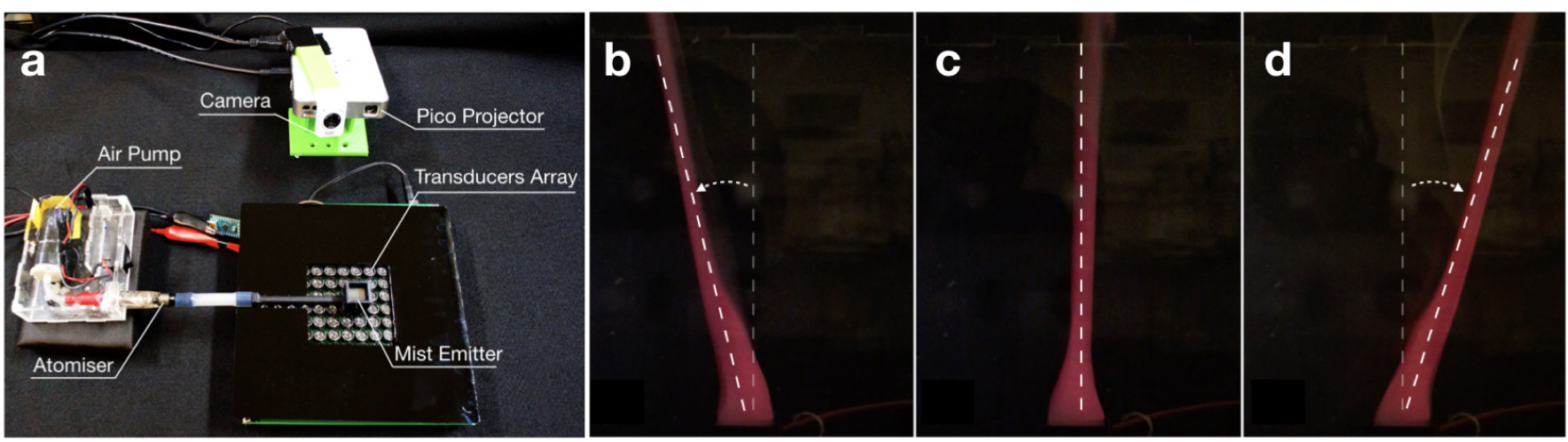

Figure 7. SonicSpray setup and example of lateral reconfiguration: (a) A SonicSpray setup consists of a phased array transducers (PAT), a 3D printed mist emitter, a mist atomiser, an air pump, a camera, and a pico projector, (b) laminar mist is steered to the left (illuminated with refracted laser beam), (c) laminar mist is steered to the center, (d) laminar mist is steered to the right.

\section{Minimum Transducers and SPL Analysis}

This part describes the identification of the minimum number of transducers and SPL required to induce airflow.

\section{Minimum transducer array required}

To find the minimum transducer array required in our proposed system, we study $R e$ and airflow speed. Our objective here is to find the smallest transducer array that satisfies two conditions: 1) it can produce airflow, and 2) the airflow is laminar. From our findings, we learn that a $6 \times 6$ array meets the objective. An array which is lower than $4 \times 4$ is not able to produce an airflow (airspeed $\sim 0 \mathrm{~m} / \mathrm{s}$ ), even though it produces laminar flow. Interestingly, Figure 8(a) shows that the SPL distribution for the $6 \times 6$ and $4 \times 4$ arrays diverges at $z \approx 42 \mathrm{~mm}$, around an SPL value of $\sim 98 \mathrm{~dB}$. This behaviour and value helped us identify $98 \mathrm{~dB}$ as the minimum SPL threshold required to induce airflow (independently of the transducer arrangement).

\section{SPL and wind correlation}

We study the correlation between SPL and airflow speed, particularly for 6x6 array. The graph in Figure 8(b) shows that SPL and airflow speed have a similar trend, peak at $60 \mathrm{~mm}$ then gradually reduce towards $140 \mathrm{~mm}$. We use the Pearson correlation coefficient method to find the relation between them. Figure 8(c) illustrates the relation. The correlation value is $r=0.98$, which implies that there is a strong correlation between SPL and airflow speed, i.e., the airflow speed increases as the SPL increases. Therefore, we suggest that to increase the airflow speed using ultrasound, one can increase the SPL (e.g. increase transducer's voltage).

\section{EVALUATION}

Here we evaluate the performance of our proposed method, and we report the lateral mist resolution by oscillation frequency.

\section{Lateral mist resolution by oscillation frequencies}

To evaluate the lateral resolution of the laminar mist, we carry out the following steps: First, we define an oscillation frequency. One complete oscillation (i.e., $1 \mathrm{~Hz}$ ) means that the reference point has moved entirely from the left $(\Delta x=-15 \mathrm{~mm})$ to the right $(\Delta x=15 \mathrm{~mm})^{1}$. Second, we continuously shift the reference point, according to the predetermined oscillation frequency, but within the displacement limit. Third, we fixed a camera $150 \mathrm{~mm}$ away from the setup, and we capture ten photos of the mist motion with 2 s exposure (e.g., see Figure 9(1)). Finally, we calculate the maximum distance of the aerosol along $z_{s}=60 \mathrm{~mm}$, denoted as (a) and (b) in the figure. We repeated these three steps, measuring oscillations of up to $25 \mathrm{~Hz}$. Then, we normalised all the distance results by the one achieved at $1 \mathrm{~Hz}$ and denoted this as lateral resolution.

Figure 9(2) illustrates the results of lateral resolution according to the oscillation frequency. We computed a regression line throughout the measured data and found that the $R^{2}=$ 0.99 , which indicates that oscillation frequency is reliable to predict the lateral resolution. The figure shows that the lateral resolution of the laminar aerosol is decreasing gradually when the oscillation frequency is increased from $1 \mathrm{~Hz}$ to $8 \mathrm{~Hz}$. The reason is that the mist particles have a time delay of $30 \mathrm{~ms}$ to adequately respond to the beam [8]. However, the laminar aerosol lateral resolution remains constant from $9 \mathrm{~Hz}$ to $25 \mathrm{~Hz}$. This finding means that the smallest lateral resolution is when lateral oscillation frequency reaches $9 \mathrm{~Hz}$.

\section{Lateral mist resolution by number of transducers}

We also evaluated the effect that reducing the numbers of transducers had on laminar mist resolution. We fixed the oscillation frequency to $5 \mathrm{~Hz}$ (changes to lateral oscillation are small at higher frequencies), recorded ten photos per array size and computed lateral mist resolution as before. Figure 9(3) shows the results of mist lateral resolution vs size of the array. The fitting is poor $\left(R^{2}=0.36<0.5\right)$, implying that even if a tendency can be observed in the data (lateral resolution increases marginally with the size of the array), it is hard to confirm such correlation, between mist behaviour and transducers number.

\section{ENABLING APPLICATIONS WITH SONICSPRAY}

SonicSpray uncovers a compact design for aerosol-based midair displays, controlled with ultrasound, allowing key benefits

\footnotetext{
${ }^{1}$ This displacement limit $(\Delta x= \pm 15 \mathrm{~mm})$ has been identified in the section "Lateral reconfiguration of the basic beam".
} 


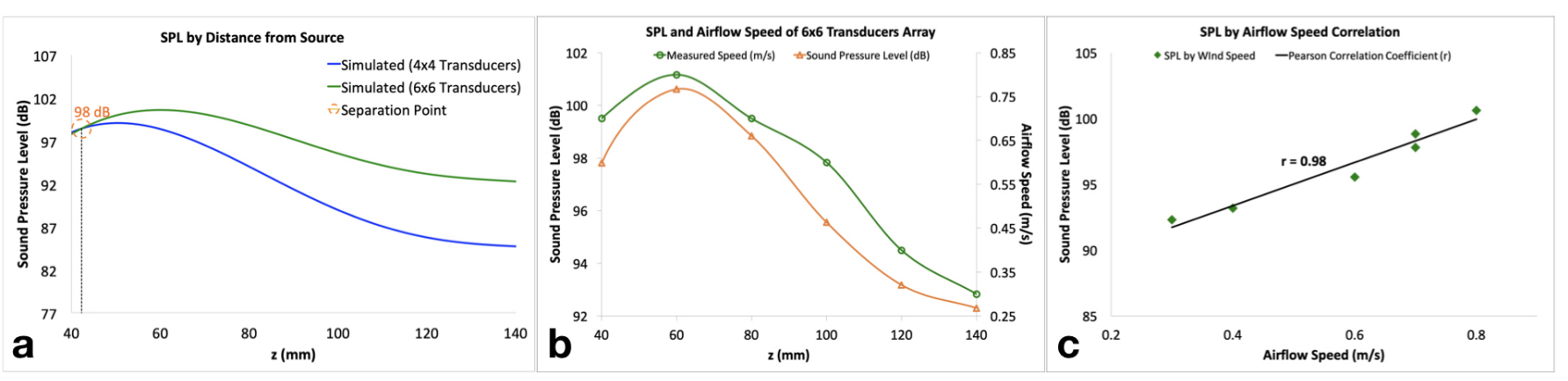

Figure 8. Graphs of Sound Pressure Level and its resulting flows: (a) The measured airflow speed by distance from transducers array. 6x6 array shows the gradual fluctuation of airflow speed while $4 \times 4$ remain constant $(0 \mathrm{~m} / \mathrm{s})$, (b) Simulated Sound Pressure Level (SPL) by distance from transducers array. SPL Separation of $6 \times 6$ and $4 \times 4$ happened at $\approx 42 \mathrm{~mm}$, (c) Graph of simulated SPL and measured airflow speed shows a similar trend, (d) A graph of correlation of simulated SPL and the measured airflow speed for 6x6 PAT.

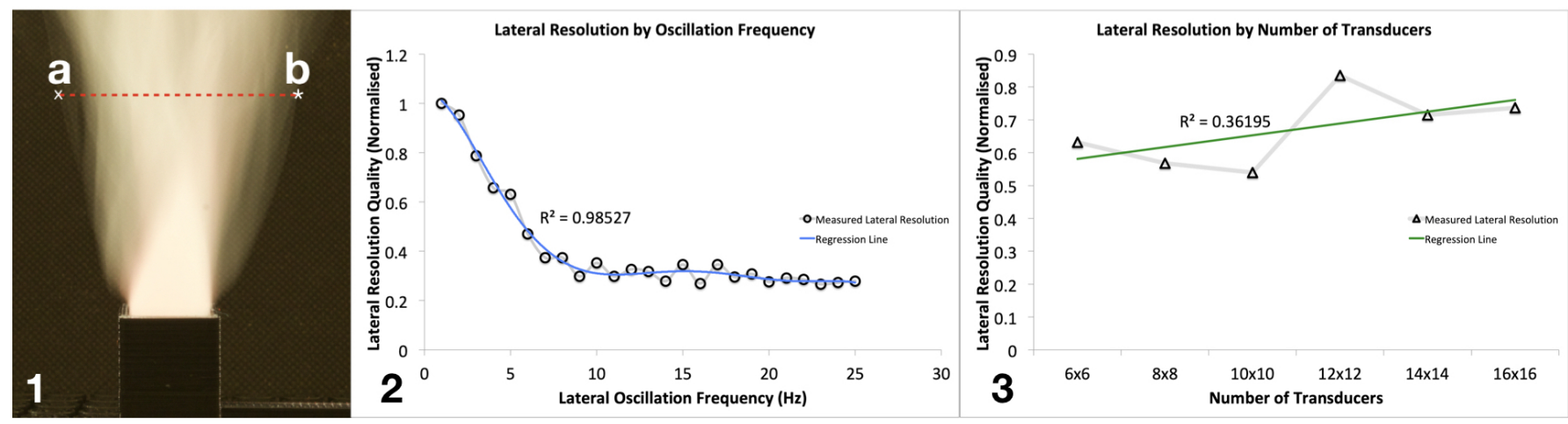

Figure 9. Lateral resolution: (1) An example photo of 2 s of long exposure to evaluate lateral resolution. The resolution is measured from (a) to (b), (2) Regression analysis of laminar mist lateral resolution with different oscillation frequencies (3) Regression analysis of laminar mist lateral resolution with a different number of transducers.

when compared to other approaches (i.e., compact, reconfigurable in 2 axes, silent, no enclosures). Although SonicSpray should be considered as a control technique, many times complementing the capabilities of other aerosol techniques, its unique features allowed us to explore novel applications even using SonicSpray as the only control technique.

By controlling the motion of a 2D stroke on $X Y$-plane, SonicSpray enables applications for learning, where a user can learn by experience. For instance, a user can touch and follow a point in 3D space, projected on the reconfigurable laminar aerosol (e.g., draw shapes or letters). We also explored a game application, for example, whack a mole game as in Figure 1(b), where the flow and acoustic pressure provided additional tactile sensations on the finger.

Another exciting application is making it as a mid-air display. We enable a reconfigurable projection that can be controlled spatially and precisely in mid-air. Figure 1(c) shows an animated butterfly is projected and sync with the reconfigured laminar mist. Such mid-air displays can also project a different type of useful contents such as a video conference (see Figure 1(d)), where a visual of a user appears in mid-air, having a conversation with the audience. The combination of these screen transformation, allows the user to form screen without the need for mechanical features (avoiding noise). Our SonicSpray precisely controls the laminar flow in mid-air and this is useful for art application (e.g., airbrush drawing) or light painting applications such in work by Huang et al. [9]. Our technique can act as an alternative to the bulky robotic arm that is used in the drawing work. SonicSPray also can be used to create small display features as in Fairy Light of Femtoseconds laser [20], while retaining a safe to touch interface.

\section{DISCUSSION}

SonicSpray offers interesting potential to produce and control laminar aerosol flows, required to create permeable mid-air displays. The technique offers clear advantages compared to other control techniques, such as avoiding moving parts (e.g., fans and mechanical actuators produce noise), allowing direct access to the display volume (e.g., avoid transparent electrodes) or allowing reconfiguration in mid-air (e.g., Mistform is limited to one axis control, and aerosol always flows vertically).

However, as a standalone technique, SonicSpray is only suited for relatively small form factors and small lateral displacements (size depends on the type of modulator -PAT or MMused). Also, the need to retain the laminarity of the flow poses limitations on the speed of the actuation achievable (although this is shared by all other approaches).

SonicSpray could also work as an excellent complement to other aerosol control approaches and form factors, such as those by Lam et al. [12, 13, 11]). For instance, by mounting Sonic Spray around the array of emitters $[13,11]$ or on the 


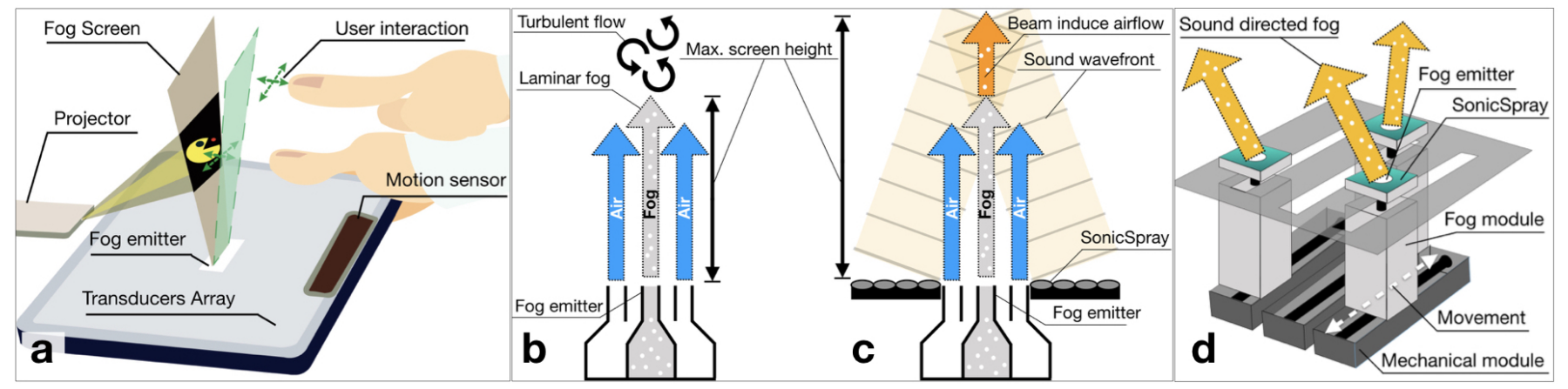

Figure 10. SonicSpray can be used as a single permeable mid-air display and can also complement other reconfiguration techniques: (a) a concept design of SonicSpray, (b) conventional FogScreens with a protective airflow degrades (slows) as it raises, and the fog display becomes turbulent, (c) SonicSpray can induce flows, even for initially static air/fog, accelerate the air of the protective flow in a FogScreen to increase their operational size, (d) SonicSpray is mounted on mechanically moving emitters to provide a wider display and large horizontal displacements.

mechanically moving emitters [12] (see Figure 10(d)), the moving emitters could be used to provide a wider display and large horizontal displacements and our technique could be used to enable further mid-air control (steer in XY plane, as fog raises).

The electrode array in [29] can only control fog in one axis (to/from the electrodes). SonicSpray could provide control along an additional axis and/or the initial direction of the flow (towards the electrodes) for further control of fog trajectories.

SonicSpray can also contribute to traditional FogScreens. FogScreens generate a laminar airflow to protect fog and retain display properties. This protective flow degrades (slows) as it raises and, when fully degraded, the fog display becomes turbulent (i.e., the degradation of protective flow limits the vertical size of the FogScreen, see Figure 10(b)). As explored in the paper, SonicSpray can induce flows of $(0.3-0.8 \mathrm{~m} / \mathrm{s})$ from low SPL levels (92-101dB), even for initially static air/fog. This could be used to continue to accelerate the air of the protective flow in a FogScreen as to increase their operational size (see Figure 10(c)).

These combinations, however, will come at the expense of sacrificing some of the benefits in SonicSpray (i.e., combined with fans, will result in noise; electrodes will result in enclosures; larger formats will require larger arrays), but they illustrate the potential of SonicSpray as an enabling technology to explore novel mid-air display formats.

\section{CONCLUSION}

This paper presented SonicSpray, a technique using steerable ultrasound Bessel beams to create and control laminar flows, with great potential as a control technique for permeable, midair displays. We described control techniques to produce such beams and explored its potential application both for PATs and hybrid modulators. We explored achievable form factors (e.g., sizes), resulting laminar flow speeds as well as the SPL levels required to create them (e.g., $0.8 \mathrm{~m} / \mathrm{s}$ at $60 \mathrm{~mm}$ and $98 \mathrm{~dB}$ ) and explored small scale applications, using SonicSpray as the sole technique (i.e., no initial airflow). We believe that SonicSpray can improve the technological feasibility of current permeable mid-air displays by enabling compact and mobile experiences. It can also act as an interesting additional control technique and enable exploration of new reconfigurable and permeable mid-air displays.

\section{ACKNOWLEDGMENTS}

This project is funded by the EU (ERC advanced grant No. 787413), the Royal Academy of Engineering through their chairs in emerging technology program, Ministry of Education, Malaysia (MoE) and Universiti Teknikal Malaysia Melaka (UTeM). We thank Luis Veloso for the images and videos.

\section{REFERENCES}

[1] Ismo Alakärppä, Elisa Jaakkola, Ashley Colley, and Jonna Häkkilä. 2017. BreathScreen: Design and Evaluation of an Ephemeral UI. In Proceedings of the 2017 CHI Conference on Human Factors in Computing Systems (CHI'17). ACM, New York, NY, USA, 4424-4429. DOI :

http://dx.doi.org/10.1145/3025453.3025973

[2] Jason Alexander, Anne Roudaut, Jürgen Steimle, Kasper Hornbæk, Miguel Bruns Alonso, Sean Follmer, and Timothy Merritt. 2018. Grand Challenges in Shape-Changing Interface Research. In Proceedings of the 2018 CHI Conference on Human Factors in Computing Systems (CHI '18). ACM, New York, NY, USA, Article 299, 14 pages. DOI : http://dx.doi.org/10.1145/3173574.3173873

[3] Tobias Alrøe, Jonas Grann, Erik Grönvall, Marianne Graves Petersen, and Jesper L. Rasmussen. 2012. Aerial Tunes: Exploring Interaction Qualities of Mid-air Displays. In Proceedings of the 7th Nordic Conference on Human-Computer Interaction: Making Sense Through Design (NordiCHI '12). ACM, New York, NY, USA, 514-523. DOI : http://dx.doi.org/10.1145/2399016.2399095

[4] Shinnosuke Ando, Kazuki Otao, Kazuki Takazawa, Yusuke Tanemura, and Yoichi Ochiai. 2017. Aerial Image on Retroreflective Particles. In SIGGRAPH Asia 2017 Posters (SA '17). ACM, New York, NY, USA, Article 7, 2 pages. DOI : http://dx.doi.org/10.1145/3145690.3145730 
[5] Rune Barnkob, Per Augustsson, Thomas Laurell, and Henrik Bruus. 2012. Acoustic radiation- and streaming-induced microparticle velocities determined by microparticle image velocimetry in an ultrasound symmetry plane. Phys. Rev. E 86 (Nov 2012), 056307. Issue 5. DOI :

http://dx.doi.org/10.1103/PhysRevE. 86.056307

[6] Euan Freeman, Julie Williamson, Sriram Subramanian, and Stephen Brewster. 2018. Point-and-Shake: Selecting from Levitating Object Displays. In Proceedings of the 2018 CHI Conference on Human Factors in Computing Systems (CHI '18). ACM, New York, NY, USA, Article 18,10 pages. DOI :

http://dx.doi.org/10.1145/3173574.3173592

[7] Keisuke Hasegawa, Liwei Qiu, Akihito Noda, Seki Inoue, and Hiroyuki Shinoda. 2017. Electronically steerable ultrasound-driven long narrow air stream. Applied Physics Letters 111, 6 (2017), 064104. DOI : http://dx.doi.org/10.1063/1.4985159

[8] K. Hasegawa, L. Qiu, and H. Shinoda. 2018. Midair Ultrasound Fragrance Rendering. IEEE Transactions on Visualization and Computer Graphics 24, 4 (April 2018), 1477-1485. DOI :

http://dx.doi.org/10.1109/TVCG.2018.2794118

[9] Yaozhun Huang, Sze-Chun Tsang, Hei-Ting Tamar Wong, and Miu-Ling Lam. 2018. Computational Light Painting and Kinetic Photography. In Proceedings of the Joint Symposium on Computational Aesthetics and Sketch-Based Interfaces and Modeling and Non-Photorealistic Animation and Rendering (Expressive '18). ACM, New York, NY, USA, Article 14, 9 pages. DOI :

http://dx.doi.org/10.1145/3229147.3229167

[10] Michinari Kono, Takayuki Hoshi, and Yasuaki Kakehi. 2014. Lapillus Bug: Creature-like Behaving Particles Based on Interactive Mid-air Acoustic Manipulation. In Proceedings of the 11th Conference on Advances in Computer Entertainment Technology (ACE'14). ACM, New York, NY, USA, Article 34, 8 pages. DOI: http://dx.doi.org/10.1145/2663806.2663850

[11] M. Lam, B. Chen, and Y. Huang. 2015. A novel volumetric display using fog emitter matrix. In 2015 IEEE International Conference on Robotics and Automation (ICRA). 4452-4457. DOI : http://dx.doi.org/10.1109/ICRA.2015.7139815

[12] M. Lam, B. Chen, K. Lam, and Y. Huang. 2014. 3D fog display using parallel linear motion platforms. In 2014 International Conference on Virtual Systems Multimedia (VSMM). 234-237. DOI :

http://dx.doi.org/10.1109/VSMM. 2014.7136689

[13] Miu-Ling Lam, Yaozhun Huang, and Bin Chen. 2015. Interactive Volumetric Fog Display. In SIGGRAPH Asia 2015 Emerging Technologies (SA '15). ACM, New York, NY, USA, Article 13, 2 pages. DOI :

http://dx.doi.org/10.1145/2818466.2818488
[14] Jinha Lee, Rehmi Post, and Hiroshi Ishii. 2011. ZeroN: Mid-air Tangible Interaction Enabled by Computer Controlled Magnetic Levitation. In Proceedings of the 24th Annual ACM Symposium on User Interface Software and Technology (UIST'11). ACM, New York, NY, USA, 327-336. DOI :

http://dx.doi.org/10.1145/2047196.2047239

[15] A. Marzo, T. Corkett, and B. W. Drinkwater. 2018. Ultraino: An Open Phased-Array System for Narrowband Airborne Ultrasound Transmission. IEEE Transactions on Ultrasonics, Ferroelectrics, and Frequency Control 65, 1 (Jan 2018), 102-111. D0I : http://dx.doi.org/10.1109/TUFFC.2017.2769399

[16] Asier Marzo, Sue Ann Seah, Bruce W. Drinkwater, Deepak Ranjan Sahoo, Benjamin Long, and Sriram Subramanian. 2015. Holographic acoustic elements for manipulation of levitated objects. Nature Communications 6 (27 Oct 2015), 8661. https://doi.org/10.1038/ncomms9661 Article.

[17] Gianluca Memoli, Mihai Caleap, Michihiro Asakawa, Deepak R. Sahoo, Bruce W. Drinkwater, and Sriram Subramanian. 2017. Metamaterial bricks and quantization of meta-surfaces. Nature Communications 8 (27 Feb 2017), 14608.

https://doi.org/10.1038/ncomms14608 Article.

[18] Mohd Adili Norasikin, Diego Martinez Plasencia, Spyros Polychronopoulos, Gianluca Memoli, Yutaka Tokuda, and Sriram Subramanian. 2018. SoundBender: Dynamic Acoustic Control Behind Obstacles. In Proceedings of the 31st Annual ACM Symposium on User Interface Software and Technology (UIST'18). ACM, New York, NY, USA, 247-259. DOI : http://dx.doi.org/10.1145/3242587.3242590

[19] Yoichi Ochiai, Takayuki Hoshi, and Jun Rekimoto. 2014. Pixie Dust: Graphics Generated by Levitated and Animated Objects in Computational Acoustic-potential Field. ACM Trans. Graph. 33, 4, Article 85 (July 2014), 13 pages. DOI :

http://dx.doi.org/10.1145/2601097.2601118

[20] Yoichi Ochiai, Kota Kumagai, Takayuki Hoshi, Jun Rekimoto, Satoshi Hasegawa, and Yoshio Hayasaki. 2016. Fairy Lights in Femtoseconds: Aerial and Volumetric Graphics Rendered by Focused Femtosecond Laser Combined with Computational Holographic Fields. ACM Trans. Graph. 35, 2, Article 17 (Feb. 2016), 14 pages. DOI : http://dx. doi.org/10.1145/2850414

[21] Themis Omirou, Asier Marzo, Sue Ann Seah, and Sriram Subramanian. 2015. LeviPath: Modular Acoustic Levitation for 3D Path Visualisations. In Proceedings of the 33rd Annual ACM Conference on Human Factors in Computing Systems (CHI'15). ACM, New York, NY, USA, 309-312. DOI :

http://dx.doi.org/10.1145/2702123.2702333 
[22] T. Omirou, A. M. Perez, S. Subramanian, and A. Roudaut. 2016. Floating charts: Data plotting using free-floating acoustically levitated representations. In 2016 IEEE Symposium on 3D User Interfaces (3DUI). IEEE, Greenville, SC, USA, 187-190. DOI : http://dx.doi.org/10.1109/3DUI.2016.7460051

[23] Kazuki Otao and Takanori Koga. 2017. Mistflow: A Fog Display for Visualization of Adaptive Shape-changing Flow. In SIGGRAPH Asia 2017 Posters (SA '17). ACM, New York, NY, USA, Article 17, 2 pages. DOI : http://dx.doi.org/10.1145/3145690.3145696

[24] Giuseppe Pesce, Giorgio Volpe, Onofrio M. Maragó, Philip H. Jones, Sylvain Gigan, Antonio Sasso, and Giovanni Volpe. 2015. Step-by-step guide to the realization of advanced optical tweezers. J. Opt. Soc. Am. B 32, 5 (May 2015), B84-B98. D0I : http://dx.doi.org/10.1364/JOSAB.32.000B84

[25] Hao Qiu, Yuki Uno, Toru Sai, Shunta Iguchi, Yota Mizutani, Takayuki Hoshi, Yoshihiro Kawahara, Yasuaki Kakehi, and Makoto Takamiya. 2018. Luciola: A Light-emitting Particle Moving in Mid-air Based on Ultrasonic Levitation and Wireless Powering. In SIGGRAPH Asia 2018 Emerging Technologies (SA '18). ACM, New York, NY, USA, Article 7, 2 pages. DOI : http://dx.doi.org/10.1145/3275476.3275479

[26] Ismo Rakkolainen. 2008. Mid-air Displays Enabling Novel User Interfaces. In Proceedings of the 1st ACM International Workshop on Semantic Ambient Media Experiences (SAME '08). ACM, New York, NY, USA, 25-30. DOI : http://dx.doi.org/10.1145/1461912 . 1461919

[27] Ismo Rakkolainen and Antti Sand. 2013. A Movable Immaterial Volumetric Display. In SIGGRAPH Asia 2013 Posters (SA '13). ACM, New York, NY, USA, Article 2, 1 pages. DOI :

http://dx.doi.org/10.1145/2542302.2542305

[28] Jaime Ruiz-Avila. 2016. Holovect: Holographic Vector Display. (Oct 2016).

https://www.kickstarter.com/projects/2029950924/ holovect-holographic-vector-display

[29] Deepak Ranjan Sahoo, Diego Martinez Plasencia, and Sriram Subramanian. 2015. Control of Non-Solid Diffusers by Electrostatic Charging. In Proceedings of the 33rd Annual ACM Conference on Human Factors in Computing Systems (CHI'15). ACM, New York, NY, USA, 11-14. DOI :

http://dx.doi.org/10.1145/2702123.2702363
[30] Deepak Ranjan Sahoo, Takuto Nakamura, Asier Marzo, Themis Omirou, Michihiro Asakawa, and Sriram Subramanian. 2016. JOLED: A Mid-air Display Based on Electrostatic Rotation of Levitated Janus Objects. In Proceedings of the 29th Annual Symposium on User Interface Software and Technology (UIST'16). ACM, New York, NY, USA, 437-448. DOI : http://dx.doi.org/10.1145/2984511.2984549

[31] P. S. Salter, Z. Iqbal, and M. J. Booth. 2013. Analysis of the Three-Dimensional Focal Positioning Capability of Adaptive Optic Elements. International Journal of Optomechatronics 7, 1 (2013), 1-14. DOI : http://dx.doi.org/10.1080/15599612.2012.758791

[32] Sue Ann Seah, Diego Martinez Plasencia, Peter D. Bennett, Abhijit Karnik, Vlad Stefan Otrocol, Jarrod Knibbe, Andy Cockburn, and Sriram Subramanian. 2014. SensaBubble: A Chrono-sensory Mid-air Display of Sight and Smell. In Proceedings of the SIGCHI Conference on Human Factors in Computing Systems (CHI '14). ACM, New York, NY, USA, 2863-2872. DOI : http://dx.doi.org/10.1145/2556288.2557087

[33] Daniel Smalley, Ting-Chung Poon, Hongyue Gao, Joshua Kvavle, and Kamran Qaderi. 2018. Volumetric Displays: Turning 3-D Inside-Out. Opt. Photon. News 29, 6 (Jun 2018), 26-33. DOI :

http://dx.doi.org/10.1364/OPN.29.6.000026

[34] Ippei Suzuki, Shuntarou Yoshimitsu, Keisuke Kawahara, Nobutaka Ito, Atushi Shinoda, Akira Ishii, Takatoshi Yoshida, and Yoichi Ochiai. 2016. Gushed Diffusers: Fast-moving, Floating, and Lightweight Midair Display. In Proceedings of the 29th Annual Symposium on User Interface Software and Technology (UIST'16 Adjunct). ACM, New York, NY, USA, 69-70. DOI : http://dx.doi.org/10.1145/2984751.2985706

[35] Yutaka Tokuda, Mohd Adili Norasikin, Sriram Subramanian, and Diego Martinez Plasencia. 2017. MistForm: Adaptive Shape Changing Fog Screens. In Proceedings of the 2017 CHI Conference on Human Factors in Computing Systems (CHI'17). ACM, New York, NY, USA, 4383-4395. DOI : http://dx.doi.org/10.1145/3025453.3025608

[36] Toshiya Yui and Tomoko Hashida. 2016. Floatio: Floating Tangible User Interface Based on Animacy Perception. In Proceedings of the 29th Annual Symposium on User Interface Software and Technology (UIST'16 Adjunct). ACM, New York, NY, USA, 43-45. DOI : http://dx.doi .org/10.1145/2984751.2985699 